\section{Atividade física e saúde: producão científica em periódicos da Edúcação Física no Brasil}

\section{Physical activity and health: production of scientific journals of Physical Education in Brazil}

Marilson Kienteka ${ }^{1,2}$

Cintia Mussi Alvim Stocchero ${ }^{3}$

Julieta Lavin Fueyo ${ }^{4}$

Romildo Luiz Monteiro Andrade ${ }^{5}$

Marcio Botelho Peixoto ${ }^{6}$

\section{RESUMO}

O principal intuito dos periódicos científicos é difundir para a comunidade científica os resultados de pesquisas por meio de suas publicações. A frequência com que o tema "Atividade Física" (AF) se faz presente nas publicações especializadas permite avaliar a consolidação da AF enquanto área da saúde no Brasil. O objetivo deste estudo foi descrever a frequência do tema da Atividade Física e Saúde (AFS) junto aos principais periódicos nacionais vinculados á área de Educação Física (EF). Este foi um estudo descritivo de screening dos termos da AFS junto aos periódicos científicos brasileiros nos anos de 2003; 2008 e 2013. Comparando as publicações da área de AFS com as demais áreas da EF, observou-se um aumento absoluto dos estudos indexados nos periódicos pesquisados (313\%) entre 2003 e 2013 . No entanto, a frequência relativa ficou estável no decênio (19,3\%), apontando também, uma evolução da produção científica de outras áreas da EF (desempenho, educação, práticas pedagógicas entre outras) nestes periódicos.

\section{PALAVRAS-CHAVE}

Atividade motora; Saúde pública; Índice de periódicos; Portais de acesso a revistas científicas.

\begin{abstract}
The main purpose of scientific journals is to disseminate to the scientific community the results of scientific research through its publications. The frequency that the topic Physical Activity (PA) is present in specialized publications evaluates the consolidation of $P A$ as the bealth area in Brazil. The aim of the study was describe the frequency of the issue of Physical Activity and Health (PAH) with leading national journals linked to the area of Physical Education (PE). This was a descriptive study, the terms of screening of PAH at Brazilian scientific journals in 2003; 2008 and 2013. Comparing the publications area of $P A H$ with other areas of PE, there was an absolute increase of studies indexed in periodicals surveyed (313\%) between 2003 and 2013, however, the relative frequency was stable in the decennary (19.3\%), also pointing an evolution of scientific production from other areas of PE (performance, education, pedagogical practices among others) in these journals.
\end{abstract}

\section{KEYWORDS}

Motor activity; Public Health; Periodical Index; Portals for Scientific fournals.
Rev Bras Ativ Fis Saúde p. 410-416

DO

http://dx.doi.org/10.12820/rbafs.v.19n4p410

1 Universidade Federal do Paraná. Programa de Pós-Graduação em Educação Física, Curitiba-PR Brasil

2 Pontifícia Universidade Católica do Paraná Escola de Saúde e Biociências. Curso de Educação Física. Grupo de Pesquisa em Atividade Física e Qualidade de Vida - GPAQ, Curitiba-PR, Brasil

3 Instituto Federal de Educação, Ciência e Tecnologia do RS, Porto Alegre, RS, Brasil 4 Centro de Investigaciones Epidemiológicas y en Servicios de Salud -CIESS. Escuela de Salud Pública, Universidad Nacional de Córdoba. Argentina.

5 Secretaria do Estadual da Saúde, Vitória, ES./5Universidade Federal do Espírito Santo, ES 6 Universidade Federal de Pelotas. Centro de Epidemiologia de Saúde Amilcar Gigante, Pelotas, $R S$ 


\section{INTRODUCCÃO}

Os periódicos científicos têm como principal objetivo, divulgar à comunidade os resultados de pesquisas científicas por meio das publicações de artigos originais, artigos de revisão, cartas ao leitor, editoriais e comentários que possam contribuir para o avanço do conhecimento em todas as áreas. De modo geral, a área da $\mathrm{AF}$, tem contribuído com a divulgação do conhecimento associados a inúmeros desfechos em saúde ${ }^{1}$.

$\mathrm{Na}$ literatura internacional, a temática da AFS ganha cada vez mais espaço, gerando uma maior disponibilidade de informações ${ }^{2}$. No Brasil, é importante destacar o crescimento do investimento público em pesquisas na área de AFS por parte do $\mathrm{CNPq}^{3}$, a evolução da pesquisa epidemiológica em AFS no Brasil ${ }^{4,5}$, o importante posicionamento da área da AFS nos Programas de Pós-graduação em EF no Brasil ${ }^{6}$ e um elevado volume anual de publicações brasileiras observadas a partir do século XXI nos periódicos internacionais ${ }^{7}$. A partir destes dados, podemos cogitar a hipótese de que houve um crescimento da temática AFS nos periódicos nacionais ao longo da última década.

No Brasil, há indício de que existe um aumento no número de estudos publicados sobre AFS, entretanto o referido estudo limitou-se a apenas um periódico $^{1}$. Sendo assim, esta pesquisa tem como objetivo verificar a participação da temática da AFS nas principais revistas da área de Educação Física no Brasil e comparar a produção científica da área em três anos distintos, 2003, 2008 e 2013.

\section{MÉTODOS}

Foi realizado um estudo descritivo, com busca de artigos publicados nos periódicos científicos brasileiros na área da EF, em três anos distintos: 2003, 2008 e 2013. Para a seleção dos periódicos foram estabelecidos alguns critérios de inclusão, primeiramente os periódicos deveriam estar na base de dado do sistema WebQualis e especificamente aqueles classificados na área de avaliação da EF no primeiro trimestre de 2014. Após a verificação dos periódicos da área específica da EF, ainda utilizou-se os seguintes critérios de inclusão: a) possuir corpo editorial e sede fixada no Brasil; b) ser classificado como A1, A2, B1 ou B2 no WebQualis; c) estar disponível on-line e d) ser específico da área, ou seja, apresentar maior parte das publicações vinculadas à área da EF. Utilizando como referencial para o último critério de inclusão, o estudo bibliométrico realizado em $2013^{8}$.

Para garantir a comparabilidade, consideramos neste estudo, a produção cientifica num intervalo de cinco anos entre cada ano revisado, elegendo assim o ano inicial o de 2003, o ano intermediário de 2008 e o ano final de 2013 por representar o último ano de indexações periódicas finalizada.

Após a definição dos periódicos, foram consultados todos os volumes e edições nos anos pesquisados, sendo considerados para análise somente os artigos originais, qualitativos ou quantitativos que contemplassem a atividade física ou aptidão física com o enfoque à manutenção ou melhoria da saúde, bem como, a qualidade de vida dos participantes independentemente se fossem apresentadas como variável dependente ou independente. Os artigos que abordaram a aptidão física aplicada ao desempenho esportivo não foram contabilizados para compor este estudo. 
Os artigos indexados nos periódicos selecionados foram classificados em duas áreas de investigação: artigos da área de AFS e outras áreas da EF. Nesse estudo os critérios utilizados para classificar os artigos como temática da área da AFS deveriam necessariamente contemplar as seguintes áreas de investigação: (a) níveis da AF e tendências temporais; (b) determinantes da prática da $\mathrm{AF}$; (c) consequências da prática da AF na saúde e (d) intervenções para promoções da AF. Os artigos originais indexados nos periódicos que atenderam o critério de inclusão nos anos selecionados foram avaliados por dois revisores, os quais realizaram a leitura dos títulos e resumos de forma independente. $\mathrm{Na}$ ocorrência de dúvida durante esta fase, foi realizada a leitura do artigo na integra. Em caso de discordância quanto à classificação da área temática, os artigos eram encaminhados para um terceiro avaliador com o objetivo de solucionar divergências desta classificação. Os dados foram tabulados em planilha e analisados utilizando-se estatística descritiva (frequências absolutas e relativas). As análises foram conduzidas no software Microsoft ${ }^{\circledR}$ Office ${ }^{\circledR}$ Excel 2010, versão para Microsoft ${ }^{\circledR}$ Windows ${ }^{\mathrm{TM}}$.

\section{RESULTADOS}

De acordo com os critérios de inclusão definidos, 11 periódicos foram selecionados para a investigação: 1) Revista Brasileira de Atividade Física \& Saúde (RBAFS) - Sociedade Brasileira de Atividade Física e Saúde; 2) Revista Brasileira de Cineantropometria \& Desempenho Humano (RBCDH) - Núcleo de Pesquisa em Cineantropometria \& Desempenho Humano da Universidade Federal de Santa Catarina; 3) Revista Brasileira de Ciência e Movimento (RBCM) - Centro de Estudos do Laboratório de Aptidão Física de São Caetano do Sul; 4) Motriz - Universidade Estadual Paulista - Rio Claro; 5) Revista da Educação Física (REF) - Escola de Educação Física da Universidade Estadual de Maringá; 6) Revista Brasileira de Ciências do Esporte (RBCE) - Colégio Brasileiro de Ciências do Esporte; 7) Revista Brasileira de Educação Física e Esporte (RBEFE) - Universidade de São Paulo; 8) Revista Movimento Escola de Educação Física da Universidade Federal do Rio Grande do Sul; 9) Revista Brasileira de Medicina do Esporte (RBME) - Sociedade Brasileira de Medicina do Exercício e do Esporte; 10) Pensar a Prática - Faculdade de Educação Física da Universidade Federal de Goiás; e 11) Licere - Programa Multidisciplinar de Mestrado em Lazer da Universidade Federal de Minas Gerais.

Entre estes periódicos selecionados, três obtiveram qualificação WebQualis A2, quatro B1 e quatro B2, sendo que apenas cinco estão indexadas no Scientific Electronic Library Online (Scielo). Ao todo 1.210 artigos originais foram identificados nas edições consultadas nos três anos. Avaliando o total de artigos originais, verificou-se um aumento no número de publicações na área da EF (200 em 2013, 389 em 2008 e 621 em 2013), assim como na subárea da AFS (38 em 2003, 77 em 2008 e 119 em 2013). Observa-se um incremento na quantidade dos artigos referidos à AFS nestes três anos selecionados para o estudo, no entanto, este aumento absoluto não se reflete na frequência relativa neste espaço de tempo. Os resultados descritivos são apresentados na Tabela 1.

$\mathrm{Na}$ classificação por área de investigação, verificou-se que os estudos com a temática "consequências da prática da $\mathrm{AF}$ na saúde" mostrou a maior proporção $(47,9 \%)$ quando comparadas as outras temáticas como "determinantes 
da prática da $\mathrm{AF}$ " (26,1\%), "níveis da AF e tendências temporais" (19,7\%) e "intervenções para promoções da AF" (6,4). Os valores absolutos e relativos podem ser observados na tabela 2 .

TABELA 1 - Demonstrativo do volume e proporção das publicações em periódicos da Educação Física que contemplaram a área da AFS nos anos de 2003, 2008 e 2013.

\begin{tabular}{|c|c|c|c|c|c|c|c|c|c|}
\hline & \multirow[b]{2}{*}{ Qualis } & \multicolumn{2}{|c|}{2003} & \multicolumn{2}{|c|}{2008} & \multicolumn{2}{|c|}{2013} & \multicolumn{2}{|c|}{2003,2008 e 2013} \\
\hline & & $\begin{array}{c}\text { Artigos } \\
\text { Totais }\end{array}$ & $\begin{array}{c}\text { Artigos } \\
\text { AFS }\end{array}$ & $\begin{array}{c}\text { Artigos } \\
\text { Totais }\end{array}$ & $\begin{array}{c}\text { Artigos } \\
\text { AFS }\end{array}$ & $\begin{array}{c}\text { Artigos } \\
\text { Totais }\end{array}$ & $\begin{array}{c}\text { Artigos } \\
\text { AFS }\end{array}$ & $\begin{array}{c}\text { Artigos } \\
\text { Totais }\end{array}$ & $\begin{array}{l}\text { Total } \\
\text { AFS }\end{array}$ \\
\hline & 2014 & $\mathrm{~N}$ & $\mathrm{~N}(\%)$ & $\mathrm{N}$ & $\mathrm{N}(\%)$ & $\mathrm{N}$ & $\mathrm{N}(\%)$ & $\mathrm{N}$ & $\mathrm{N}(\%)$ \\
\hline RBAFS & B2 & 13 & $7(53,8)$ & 16 & $13(81,3)$ & 60 & $37(61,7)$ & 89 & $57(64,0)$ \\
\hline RBCDH & B1 & 14 & $6(42,9)$ & 53 & $6(11,3)$ & 68 & $25(36,8)$ & 135 & $42(31,1)$ \\
\hline RBME & A2 & 17 & $6(35,3)$ & 74 & $18(24,3)$ & 88 & $18(20,5)$ & 179 & $42(23,5)$ \\
\hline RBCM & B2 & 44 & $10(22,7)$ & 35 & $8(22,9)$ & 51 & $12(23,5)$ & 130 & $30(23,1)$ \\
\hline REF & B1 & 10 & $2(20,0)$ & 52 & $17(32,7)$ & 52 & $6(11,5)$ & 114 & $25(21,9)$ \\
\hline RBEFE & B1 & 14 & $5(35,7)$ & 28 & $5(17,9)$ & 41 & $8(19,5)$ & 83 & $18(21,7)$ \\
\hline MOTRIZ & A2 & 20 & $0(0,0)$ & 38 & $4(10,5)$ & 75 & $8(10,7)$ & 133 & $12(9,0)$ \\
\hline MOVIMENTO & A2 & 12 & $1(8,3)$ & 18 & $1(5,6)$ & 44 & $1(2,3)$ & 74 & $3(4,1)$ \\
\hline RBCE & B1 & 37 & $1(2,7)$ & 37 & $0(0,0)$ & 61 & $3(4,9)$ & 135 & $4(3,0)$ \\
\hline $\begin{array}{l}\text { PENSAR A } \\
\text { PRÁTICA }\end{array}$ & B2 & 9 & $0(0,0)$ & 16 & $0(0,0)$ & 59 & $1(1,7)$ & 84 & $1(1,0)$ \\
\hline \multirow[t]{2}{*}{ LICERE } & B2 & 10 & $0(0,0)$ & 22 & $0(0,0)$ & 32 & $0(0,0)$ & 64 & $0(0,0)$ \\
\hline & Total & 200 & $38(19,0)$ & 389 & $77(19,8)$ & 621 & $119(19,2)$ & 1210 & $234(19,3)$ \\
\hline
\end{tabular}

AFS: Atividade Física e Saúde; RBAFS: Revista Brasileira de Atividade Física e Saúde; RBCDH: Revista Brasileira de Cineantropometria \& Desempenho Humano; RBCM: Revista Brasileira de Ciência e Movimento; RBME: Revista Brasileira de Medicina do Esporte; MOTRIZ: Motriz - Universidade Estadual Paulista; REF: Revista da Educação Física; RBCE: Revista Brasileira de Ciências do Esporte; RBEFE: Revista Brasileira de Educação Física e Esporte; MOVIMENTO: Revista Movimento; RBME: Revista Brasileira de Medicina do Esporte; PENSAR A PRÁTICA: Faculdade de Educação Física da Universidade Federal de Goiás; LICERE: Programa Multidisciplinar de Mestrado em Lazer da Universidade Federal de Minas Gerais.

TABELA 2 - Áreas temáticas de investigação das publicações em periódicos da Educação Física que contemplaram AFS nos anos de 2003, 2008 e 2013.

\begin{tabular}{lcccccccccc}
\hline & \multicolumn{2}{c}{2003} & \multicolumn{2}{c}{2008} & \multicolumn{2}{c}{2013} & \multicolumn{2}{c}{ Total } \\
\hline & $\mathrm{N}$ & $\%$ & $\mathrm{~N}$ & $\%$ & $\mathrm{~N}$ & $\%$ & $\mathrm{~N}$ & $\%$ \\
\hline Consequências da prática da AF na saúde & 17 & 44,8 & 38 & 49,5 & 57 & 47,9 & 112 & 47,9 \\
\hline Determinantes da prática da AF & 9 & 23,7 & 21 & 27,3 & 31 & 25,6 & 61 & 26,1 \\
\hline Níveis da AF e tendências temporais & 9 & 23,7 & 12 & 15,6 & 25 & 21,4 & 46 & 19,7 \\
\hline Intervenções para promoções da AF & 3 & 7,9 & 6 & 7,8 & 6 & 5,1 & 15 & 6,4 \\
\hline Total & 38 & 100 & 77 & 100 & 119 & 100 & 234 & 100 \\
\hline
\end{tabular}

A RBAFS foi o periódico que mais publicou na área da AFS na somatória dos três anos avaliados, apresentando um crescimento no volume de publicações nesta área. No entanto, apesar de mostrar um acréscimo de 1,9 vezes no volume de publicação de 2003 para 2008 e 2,8 vezes de 2008 para 2013, a proporção de $2013(81,3 \%)$ ficou menor do que a observada anteriormente em 2008 (61,7\%), quando comparada com o montante geral de publicações 
na área de EF. Outras revistas que apresentaram destaque no volume geral de publicação de estudos da área da AFS nestes três anos foram a RBCDH e RBME. Por outro lado, as Revistas, Movimento, RBCE, Pensar a Prática e Licere apresentaram poucas $(<5)$ ou nenhuma publicação de estudos com ênfase na área da AFS nos anos estudos. A comparação das proporções das publicações na área de AFS pode ser contemplada na Figura 1.

Quanto à classificação dos periódicos brasileiros pelo sistema WebQualis, a publicação científica da área da AFS em periódicos do estrato A2 (classificação de 03/2014) mostrou uma pequena diminuição entre 2003 e 2013 (de 14,3\% para 13,0\%, respectivamente). Já nas publicações dos outros estratos, observou-se uma estabilidade no WebQualis B1 e um aumento no WebQualis B2. Estes dados podem ser observados na Figura 2.

FIGURA 1 - Proporção de publicações nos periódicos brasileiros focadas na temática "Atividade Física e Saúde" em 2003, 2008 e 2013.

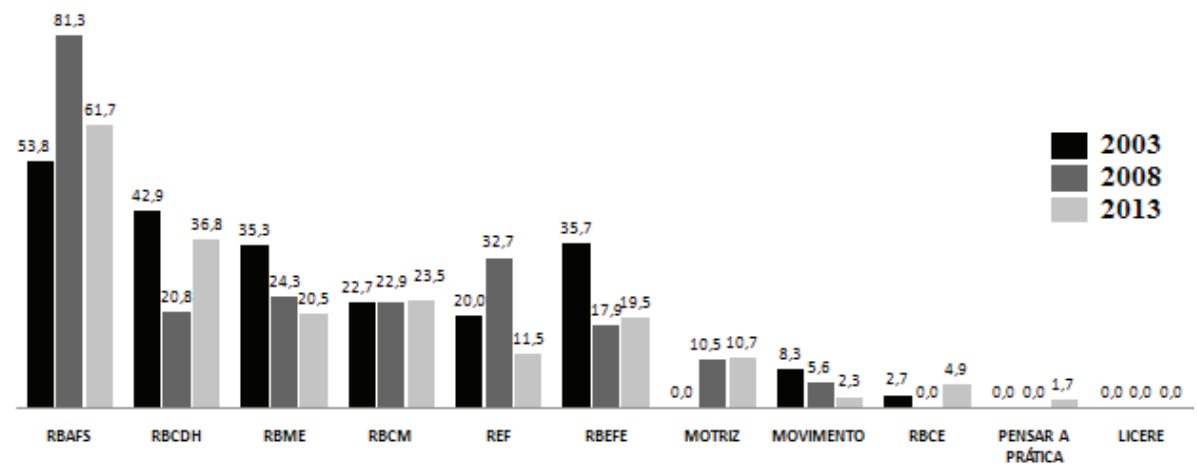

FIGURA 2 - Publicações nas revistas brasileiras focadas na atividade física e saúde comparada com outras áreas da educação física nos anos de 2003, 2008 e 2013.

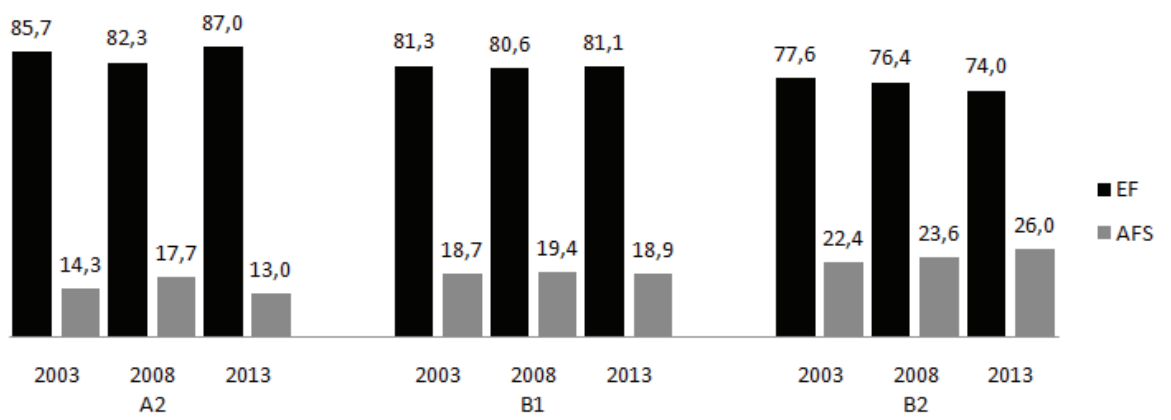

\section{DISCUSSÃO}

O principal achado deste estudo é que apesar do aumento no número absoluto de artigos originais publicados na área de AFS, constatou-se que não houve um aumento relativo nos artigos publicados que abordaram a temática AFS, dentro dos principais periódicos da área da EF. Esta ocorrência se dá ao fato do crescimento geral da publicação da área da EF como um todo, demonstrando a consolidação da mesma como área do conhecimento.

Dados relativos ao triênio de 1998-2000 revelam que a pós-graduação em EF produziu um total de 507 artigos $^{9}$. Em nossa pesquisa encontramos 1.210 
artigos originais publicados na área de EF na somatória dos anos avaliados, sendo que exclusivamente no ano de 2013 foram publicados 621 artigos. Esses dados evidenciam que a produção científica na área de EF aumentou ao longo dos anos, corroborando com os nossos achados. Segundo um estudo publicado por Molina Neto (2006), a área de AFS foi a que apresentou o maior número de teses defendidas no período de 2001 a $2005^{10}$.

No presente estudo, os periódicos que apresentaram maior contribuição na área de AFS foram a RBAFS, RBCDH, RBME e RBCM, sendo que um estudo previamente citado, já indicava esse aumento no número de artigos publicados na RBAFS, principalmente a partir de 2007, resultado de um maior número de submissões na revista ${ }^{1}$.

O crescimento absoluto nas publicações nacionais encontrado neste estudo se relaciona diretamente com o surgimento de grupos de pesquisa na área da AFS no Brasil e o aumento da oferta dos cursos de Pós-graduação com linhas de pesquisa em $\mathrm{AFS}^{6,11}$, além do crescimento de financiamentos pelo $\mathrm{CNPq}$ de projetos e bolsas de produtividade nas linhas de pesquisa em $\mathrm{AFS}^{3}$. No entanto, o fato de não apontado aumento proporcional das publicações a nível nacional, apesar de todo o investimento e crescimento da área já relatado ${ }^{3}$, pode ser explicado por vários motivos, por exemplo, a possibilidade de investigadores da área publicarem em periódicos de menor impacto que não foram contemplados nesta pesquisa, assim como uma possível opção por periódicos especificamente da área da saúde e epidemiologia. E certamente, um fator que merece consideração é a possível escolha por periódicos internacionais para publicação, visto seu maior prestígio e maior impacto frente à comunidade científica, já que são poucos os periódicos nacionais da EF que estão indexados na base de dados on-line da Scielo, o que diminui o alcance dos resultados destas publicações.

Entre as limitações do estudo, podemos mencionar o pouco detalhamento na classificação, impedindo a avaliação das outras áreas da EF. Ainda, a restrição feita em nossa pesquisa aos periódicos da área de EF, excluindo, portanto, publicações de outros periódicos da área da saúde que poderiam englobar a AFS, por exemplo, periódicos da Nutrição, Fisioterapia e Terapia Ocupacional. Além disso, não foram avaliados os periódicos internacionais, assim como aqueles com classificação WebQualis B3 e subsequente. Outra limitação deste estudo foi à escolha de períodos com intervalo de cinco anos, não apontando assim, a evolução absoluta das publicações destas revistas ano após ano.

Portanto, conclui-se pelo presente estudo um aumento absoluto dos estudos focados na área da AFS indexados nos periódicos pesquisados, no entanto a frequência relativa ficou estável na comparação entre os anos estudados, apontando uma evolução da publicação de outras áreas da EF nos periódicos brasileiros. A área temática "consequências da prática da AF na saúde" apresentou a maior proporção entre as publicações analisadas. Futuros estudos poderão abordar publicações de outros anos, observando um escopo maior de revistas da área da saúde, bem como, incluir os estudos nacionais publicados em periódicos internacionais.

\section{REFERÊNCIAS}

1. Borges TT, Corrêa LQ, Freitas MP, Silva MC. Análise da história da Revista Brasileira de Atividade Física e Saúde: 1995-2012. Rev Bras de Ativ Fís Saúde. 2013;18(3):371-7. 
2. Haskell WL, Blair SN, Hill JO. Physical activity: health outcomes and importance for public health policy. Prev Med. 2009;49(4):280-2.

3. Silva ICMD, Santin-Medeiros F, Bertapelli F, Coelho APS, Silva SGD. Pesquisa em atividade física e saúde no Brasil: um recorte dos investimentos em projetos e bolsas de produtividade CNPq. Rev Bras de Ativ Fís Saúde. 2014;19(3):325-32.

4. Hallal PC, Dumith SdC, Bastos JP, Reichert FF, Siqueira FV, Azevedo MR. Evolução da pesquisa epidemiológica em atividade física no Brasil: revisão sistemática. Rev de Saúde Pública. 2007;41:453-60.

5. Ramires VV, Becker LA, Sadovsky ADI, Zago AM, Bielemann RM, Guerra PH. Evolução da pesquisa epidemiológica em atividade física no Brasil: Atualização de uma revisão sistemática. Rev Bras de Ativ Fís Saúde. 2014;(prelo).

6. Garcia LMT, Böhm AW, Bacil EDA, Cruz MFd, Santo RCdE. A inserção da subárea de Atividade Física e Saúde nos programas de pós-graduação em Educação Física no Brasil. Rev Bras de Ativ Fís Saúde. 2014;19(2):215-22.

7. Cordeiro DSBC, Rey-López JP, Hartwig FP, Silva TGBe, Bielemann RM, Coll CVN. A contribuição do Brasil para a pesquisa em atividade física e saúde no cenário internacional. Rev Bras de Ativ Fís Saúde. 2014;(prelo).

8. Job I. Gestão editorial das revistas brasileiras do campo de educação física e ciência do esporte. Porto Alegre: Programa de Pós-graduação em ciências do movimento humano, Universidade Federal do Rio Grande do Sul; 2013.

9. Erdmann AL, Fernandes JD. Publicações científicas qualificadas na Enfermagem Brasileira. Revista Brasileira de Enfermagem. 2009;62(4):499-501.

10. Molina VN, Günther MCC, Bossle F, Wittizoreck ES, Molina RMK. Reflexões sobre a produção do conhencimento em educação física e ciência do esporte. Rev Bras de Ciência do Esporte. 2008;28(1).

11. Quadros HMd, Afonso MdR, Ribeiro JAB. O Cenário da Pós-Graduação em Educação Física: Contextos e possibilidades na região sul do Brasil. Rev Bras de Ativ Fís Saúde. 2013;18(5):576-84.

\section{ENDERECTO PARA}

CORRESPONDÊNCIA

MARILSON KIENTEKA

Pontifícia Universidade Católica do

Paraná - PUCPR. Escola de Saúde e

Biociência - Curso de Educação Física

Grupo de Pesquisa em Atividade Física e

Qualidade de Vida - GPAQ. Rua Imaculada

Conceição, 1155 - Prado Velho - CEP

80215-901 - Curitiba-PR, Brasil

E-mail: marilson_kientekaßahotmail.com 